\title{
Spin-Polarized Transport in Inhomogeneous Magnetic Semiconductors: Theory of Magnetic/Nonmagnetic $\boldsymbol{p}$ - $n$ Junctions
}

\author{
Igor Žutić, ${ }^{1}$ Jaroslav Fabian, ${ }^{1,2, *}$ and S. Das Sarma ${ }^{1}$ \\ ${ }^{1}$ Department of Physics, University of Maryland at College Park, College Park, Maryland 20742-4111 \\ ${ }^{2}$ Max-Planck Institute for the Physics of Complex Systems, D-01187 Dresden, Germany
}

(Received 5 June 2001; published 29 January 2002)

\begin{abstract}
A theory of spin-polarized transport in inhomogeneous magnetic semiconductors is developed and applied to magnetic/nonmagnetic $p-n$ junctions. Several phenomena with possible spintronic applications are predicted, including spin-voltaic effect, spin valve effect, exponential and giant magnetoresistance. It is demonstrated that only nonequilibrium spin can be injected across the space-charge region of a $p-n$ junction, so that there is no spin injection (or extraction) at low bias.
\end{abstract}

DOI: $10.1103 /$ PhysRevLett.88.066603

Semiconductor spintronics is an emerging field promising device applications with more functionality and better integrability with traditional semiconductor technology than metal-based spintronics [1]. Spin injection into a semiconductor has been demonstrated [2,3], but an important question, what this injected spin is useful for, remains largely unanswered (see, however, [4,5]). This Letter formulates the problem of spin and charge bipolar (electron and hole) transport in inhomogeneously doped magnetic semiconductors (such as CdMnSe or GaMnAs, whose carrier $g$ factors are large due to magnetic doping). In particular, we show that a magnetic/nonmagnetic $p-n$ junction has large magnetoresistance and displays spinvoltaic and spin valve effects: a current, whose direction changes with the direction of magnetic field, can flow without any applied bias. These effects should be useful for sensing magnetic fields, and for probing spin polarization and spin relaxation.

To introduce the equations for spin and charge bipolar transport in inhomogeneously doped magnetic semiconductors, consider a semiconductor doped with $N_{a}(\mathbf{r})$ acceptors and $N_{d}(\mathbf{r})$ donors, and with magnetic impurities, whose density varies in space and whose presence leads to large $g$ factors for electrons and holes, $g_{n}(\mathbf{r})$ and $g_{p}(\mathbf{r})$. In a homogeneous magnetic field $B$ the carrier energies are Zeeman split: spin up $(\lambda=1$ or $\uparrow)$ and spin down $(\lambda=-1$ or $\downarrow)$ electrons have their energy shifted by $-\lambda q \zeta_{n}(\mathbf{r})=-\lambda g_{n}(\mathbf{r}) \mu_{B} B / 2$, where $\mu_{B}$ is Bohr magneton and $q$ is the proton charge; the energy of holes changes by $\lambda q \zeta_{p}(\mathbf{r})=\lambda g_{p}(\mathbf{r}) \mu_{B} B / 2$. Carrier charge current densities, resulting from the electric field $\mathbf{E}=-\nabla \phi$, nonuniform magnetic "potentials" $\zeta$, electron (hole) densities $c=n(c=p)$, are

$$
\mathbf{J}_{c \lambda}=q \mu_{c \lambda} c_{\lambda} \mathbf{E} \pm q D_{c \lambda} \nabla c_{\lambda}-q \lambda \mu_{c \lambda} c_{\lambda} \nabla \zeta_{c},
$$

where the upper (lower) sign is for electrons (holes), $\mu$ and $D$ stand for mobility and diffusivity, and the "magnetic" drift $q \nabla \zeta$ forces carriers with opposite spins to go in opposite directions. Equation (1) follows from the requirement that carrier densities in an inhomogeneous environment have the quasiequilibrium form [6]. From Eq. (1)
PACS numbers: 72.25.Dc, 72.25.Mk

the carrier charge and spin current densities $J=J_{\uparrow}+J_{\downarrow}$ and $J_{s}=J_{\uparrow}-J_{\downarrow}$ are

$$
\begin{gathered}
\mathbf{J}_{c}=\sigma_{c} \mathbf{E}-\sigma_{s c} \nabla \zeta_{c} \pm q D_{c} \nabla n \pm q D_{s c} \nabla s_{c}, \\
\mathbf{J}_{s c}=\sigma_{s c} \mathbf{E}-\sigma_{c} \nabla \zeta_{c} \pm q D_{s c} \nabla c \pm q D_{c} \nabla s_{c} .
\end{gathered}
$$

Here $c=c_{\uparrow}+c_{\downarrow}$ and $s_{c}=c_{\uparrow}-c_{\downarrow}$, and we introduced carrier charge and spin conductivities $\sigma_{c}=q\left(\mu_{c} c+\right.$ $\left.\mu_{s c} s_{c}\right)$ and $\sigma_{s c}=q\left(\mu_{s c} c+\mu_{c} s_{c}\right)$, where $\mu_{c}=\left(\mu_{c \uparrow}+\right.$ $\left.\mu_{c \downarrow}\right) / 2$ and $\mu_{s c}=\left(\mu_{c \uparrow}-\mu_{c \downarrow}\right) / 2$, and similarly for diffusivities. Equation (2), which is a generalization of the Johnson-Silsbee magnetotransport equations [7], reflects the spin-charge coupling in bipolar transport in inhomogeneous magnetic semiconductors: a spatial variation in spin density, as well as in $q \nabla \zeta$, can cause charge currents, while spin currents can flow as a result of a spatial variation of carrier densities and $\phi$.

Generation and recombination of electrons and holes are assumed to be mostly due to band-to-band processes. Furthermore, electrons (holes) with a given spin are assumed to recombine with holes (electrons) of either spin. The stationary continuity equation for carriers is

$$
\nabla \cdot \frac{\mathbf{J}_{c \lambda}}{q}= \pm w_{c \lambda}\left(c_{\lambda} \bar{c}-c_{\lambda 0} \bar{c}_{0}\right) \pm \frac{c_{\lambda}-c_{-\lambda}-\lambda \tilde{s}_{c}}{2 T_{1 c}} .
$$

Here $\bar{c}$ is $p(n)$ if $c$ is $n(p), c_{0}$ is the equilibrium carrier density, $w$ is the (generally spin dependent) band-to-band recombination rate, and $T_{1}$ is the spin relaxation time. Spin relaxation equilibrates carrier spins while preserving nonequilibrium carrier density, so, for a nondegenerate semiconductor, $\tilde{s}_{c}=\alpha_{c 0} c$, where $\alpha_{c 0}=s_{c 0} / c_{0}=$ $\tanh \left(\zeta_{c} / V_{T}\right)$ is the equilibrium carrier spin polarization $\left(V_{T}=k_{B} T / q\right.$, with $k_{B}$ being the Boltzmann constant and $T$ temperature). Equations (1) [or (2) and (3)], (4), and Poisson's equation $\nabla \cdot \epsilon \mathbf{E}=\rho$, where $\rho=q(p-n+$ $N_{d}-N_{a}$ ) and $\epsilon$ is the dielectric constant of the semiconductor, determine the distributions of charge and spin in a magnetic semiconductor under applied bias $\mathrm{V}$.

We have made a number of simplifying assumptions in our theory which are not strictly valid in real magnetic 
semiconductors. Most importantly, we treat carrier states as spin doublets and neglect both the effect of magnetic impurities on the band structure and the response of the magnetic ions to the magnetic field of polarized carriers [8]. Our formalism can easily be tailored to include these more complicated effects on a case-by-case basis. Our approach is to consider a simple but reasonable model to illustrate new phenomena that can occur in inhomogeneous magnetic semiconductors.

We now apply the above theory to the problem of a magnetic/nonmagnetic $p$ - $n$ junction with nondegenerate carrier densities and with only electrons magnetically active $\left(\zeta_{p}=0\right)$. Two cases are considered (Fig. 1): (i) the magnetic $n$ side to study spin injection, and (ii) the magnetic $p$ side, to study spin extraction and magnetoresistance phenomena. We assume perfect Ohmic contacts (both carriers and spins at equilibrium) as boundary conditions, except when we study the dependence of the current on nonequilibrium spin, in which case we keep the ohmic contacts only for carrier densities.

Specifically we consider an $L=12 \mu \mathrm{m}$ long GaAs $p$ - $n$ junction at room temperature, doped with $N_{a}=3 \times$ $10^{15} \mathrm{~cm}^{-3}$ acceptors to the left, $N_{d}=5 \times 10^{15} \mathrm{~cm}^{-3}$ donors to the right [5], and with magnetic impurities inducing electronic $g$ factor which follows the shape of $N_{a}$ $\left(N_{d}\right)$ for the magnetic $p(n)$ region. Holes are unpolarized, so we omit the label $n$ when dealing with spin-related quantities, and reserve that symbol for the $n$-region-related variables. Figure 1 depicts our geometry and the distribution of electrons and holes for various cases considered below. The materials parameters are the electron and hole diffusivities, $D_{n}=10 D_{p}=103.6 \mathrm{~cm}^{2} \mathrm{~s}^{-1}$, and mo-

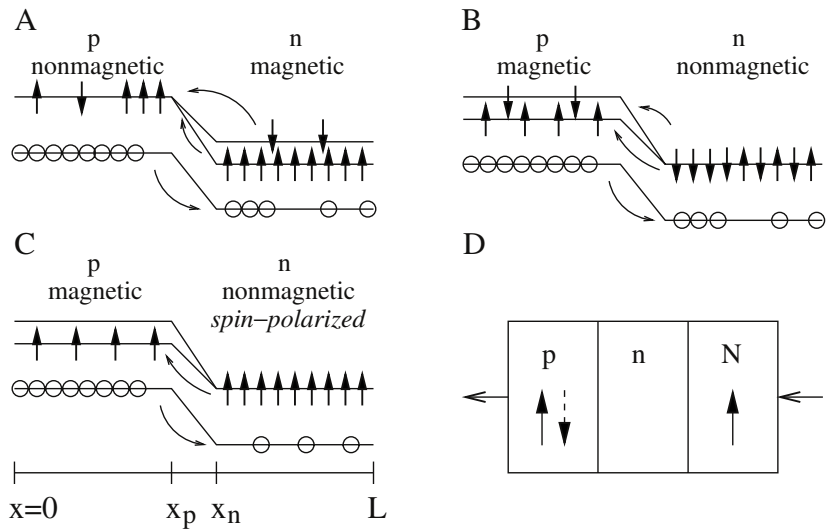

FIG. 1. Band-energy schemes for magnetic/nonmagnetic $p-n$ junctions with magnetically active electrons (arrows). Holes (circles) are unpolarized. (A) Electrons from the magnetic $n$ region (note the band split) can be injected into the nonmagnetic $p$ region only at large bias. (B) Similarly for spin extraction if the $p$ region is magnetic. (C) If the $p$ region is magnetic and there is a nonequilibrium spin in the $n$ region, a giant magnetoresistance and spin-voltaic effects arise. The former can be observed in a scheme (D), where electron spin is injected from a magnetic $N$ (through a heterostructure) into the nonmagnetic $n$ region ( $p$ is magnetic). bilities $\mu_{n}=10 \mu_{p}=4000 \mathrm{~cm}^{2} \mathrm{~V}^{-1} \mathrm{~s}^{-1}$. The intrinsic (nonmagnetic) carrier density is $n_{i}=1.8 \times 10^{6} \mathrm{~cm}^{-3}$, the dielectric constant $\epsilon=13.1$ of the vacuum permittivity; the calculated built-in voltage is $1.1 \mathrm{~V}$ at $B=0$. Recombination rate $w=(1 / 3) \times 10^{-5} \mathrm{~cm}^{3} \mathrm{~s}^{-1}$, and the spin relaxation time $T_{1}=0.2 \mathrm{~ns}$. The minority [9] diffusion lengths are [5] $L_{n}=1 \mu \mathrm{m}, L_{p}=0.25 \mu \mathrm{m}$, and the electron spin diffusion length in the $n(p)$ region is $L_{s n}=$ $1.4 \mu \mathrm{m}\left(L_{s p}=0.8 \mu \mathrm{m}\right)$. Figure 2 illustrates the doping profile and a typical profile of carrier and spin densities.

We first ask the important question whether spin can be injected and extracted into/from the nonmagnetic region. Figure 3 shows the results of our numerical calculations, where we plot spin (density, not current) polarization $\alpha=s / n$. At small bias (below the built-in value) there is no significant spin injection or extraction. As the bias increases, the injection and extraction become large and intensify with increasing $T_{1}$. The reason why there is no spin injection or extraction at small bias is that although there are exponentially more, say, spin up than spin down electrons in the magnetic side, the barrier for crossing the space-charge region is exponentially larger for spin up than for spin down electrons (see Fig. 1). Those two exponential effects cancel out, leaving no net spin current flowing through the space-charge region. At large bias (where numerics is indispensable) spin injection is possible, a result of building up a nonequilibrium spin at the spacecharge region.

The current through a magnetic/nonmagnetic $p-n$ junction depends on magnetic field. This dependence has two sources. First, a magnetic field makes the band gap spin dependent, leading to the increased density of the minority carriers. For example, the equilibrium density of electrons

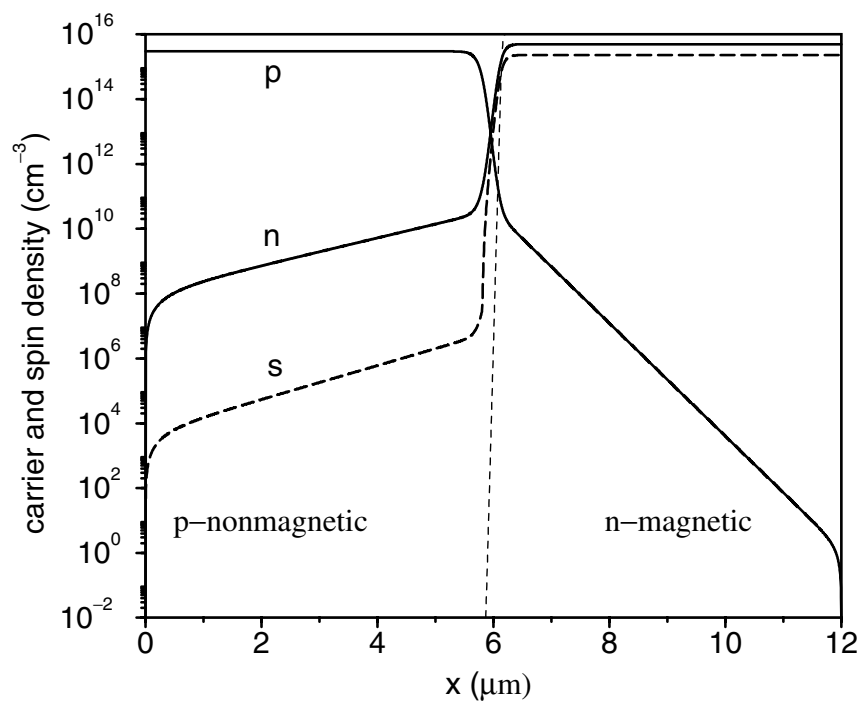

FIG. 2. Calculated electron $(n)$, hole $(p)$, and spin $(s)$ density profiles for a magnetic $p-n$ junction with $q \zeta=0.5 k_{B} T$ in the (magnetic) $n$ region, under forward applied bias of $V=0.8 \mathrm{~V}$. The thin dashed line indicates the doping profile $N_{d}-N_{a}$ (not to scale). 


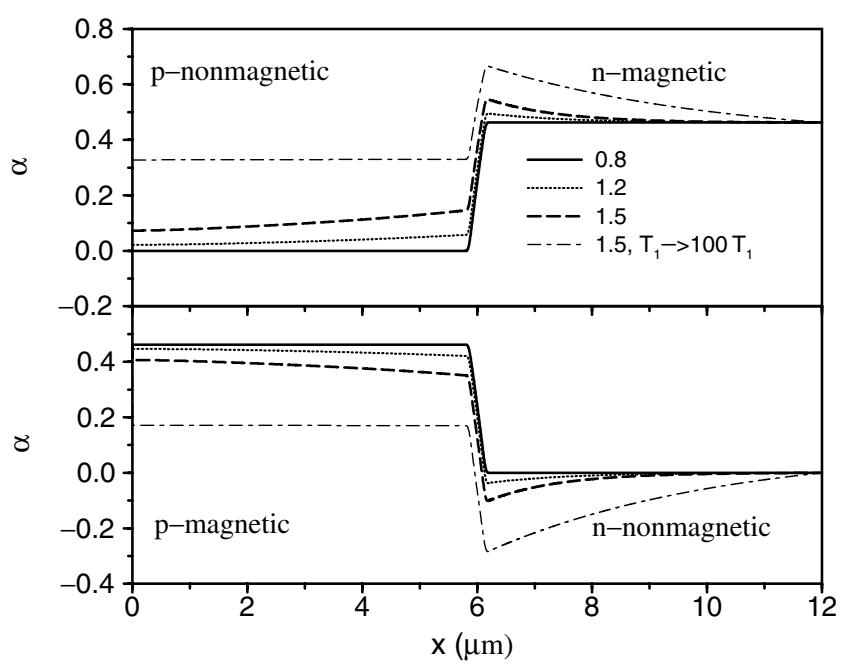

FIG. 3. Calculated spin polarization profiles for different forward bias and spin relaxation rates. Spin injection (top) from the magnetic $n$ side into the nonmagnetic $p$ side occurs only at large bias (indicated by the numbers in volts). The largest injection in the graph is for $V=1.5 \mathrm{~V}$, with spin relaxation time of $100 T_{1}$. Similar behavior is observed for spin extraction from the nonmagnetic $n$ region into the magnetic $p$ region (bottom). The magnetic splitting is $q \zeta=0.5 k_{B} T$.

in a magnetic $p$ region (Fig. 1C) is $n_{0}=n_{i}^{2} \cosh (\zeta /$ $\left.V_{T}\right) / N_{a}$ [in equilibrium, $\left.n_{0} p_{0}=n_{i}^{2} \cosh \left(\zeta / V_{T}\right)\right]$. The current through a $p-n$ junction is proportional to the density of the minority (not majority) carriers, and so it will grow exponentially with $B$ for $\zeta \geqslant V_{T}$. The exponential magnetoresistance for the system in Fig. 1B is shown in Fig. 4. The effect diminishes with increasing bias. The second way a current can depend on $B$ is if a nonequilibrium spin with polarization $\alpha(L)$ is introduced (optically or by electrical spin injection) into the sample (Figs. 1C and 1D). The exponential magnetoresistance becomes giant (meaning, the resistance changes when the orientation of the magnetic moment in the magnetic region with respect to the orientation of the injected spin changes) as seen in Fig. 4. The current is exponentially sensitive to both $B$ and $\alpha(L)$, being large (small) if they have the same (opposite) sign.

To explain analytically our numerical results, we generalize the Shockley approximation [6] for the $p-n$ junction to include nonequilibrium spin, which is given, in the quasineutral regions, by the spin-diffusion equation

$$
D_{n} \nabla^{2} s=w\left(s p-s_{0} p_{0}\right)+(s-\tilde{s}) / T_{1},
$$

where $\tilde{s}=\alpha_{0} n$, of which $n$ is the solution of the corresponding carrier diffusion equation. We consider the magnetic $p$ region, and impose a nonequilibrium spin polarization $\alpha(L)$ at the right boundary (see Fig. 1C, where also the boundary points used below are indicated). We postulate as the second boundary condition for spin, in the spirit of the quasiequilibrium approximation [6], that spin
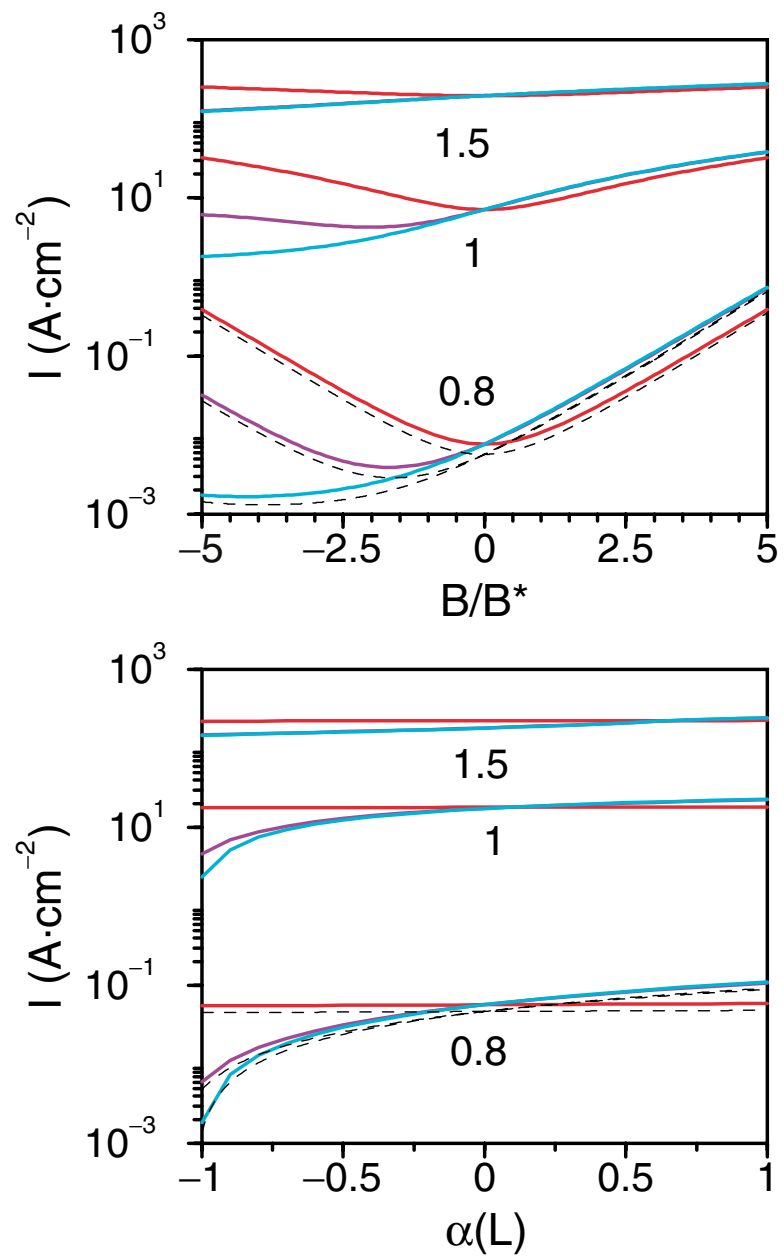

FIG. 4 (color). Calculated $I-B$ (top) and $I-\alpha$ (bottom) characteristics for the magnetic $p-n$ junction with the magnetic $p$ region, under different bias (indicated in volts). We use $B^{*}=2 k_{B} T / g \mu_{B}(\approx 900 / g$ Tesla at room temperature $)$ as the scale for magnetic field $\left(B / B^{*}=q \zeta / V_{T}\right)$. In the top graph the red line is for $\alpha(L)=0$, and the violet (cyan) line for $\alpha=1$ and spin relaxation time of $100\left(10^{4}\right) T_{1}$. The bottom graph is for $B / B^{*}=3$, and the red, violet, and cyan lines are for the spin relaxation times of $T_{1}, 100 T_{1}$, and $10^{4} T_{1}$. The dashed lines are the analytical calculations. In both graphs, at $V=1.5 \mathrm{~V}$, lines for $100 T_{1}$ and $10^{4} T_{1}$ almost coincide.

current vanishes at $x_{n}$, the $n$-side boundary of the spacecharge region. The spin polarization in the $n$ region is

$$
\alpha(x)=\alpha(L)\left[\cosh (\eta)-\tanh \left(\eta_{n}\right) \sinh (\eta)\right],
$$

where $\eta=(L-x) / L_{s n}$ and $\eta_{n}=\left(L-x_{n}\right) / L_{s n}$. At $x=x_{n}, \quad \alpha_{n} \equiv \alpha\left(x_{n}\right)=\alpha(L) / \cosh \left[\left(L-x_{n}\right) / L_{s n}\right] . \quad$ In the $p$ region $\alpha(0)=\alpha_{0}$ (Ohmic boundary condition for spin) and the second boundary condition, that at $x=x_{p}$, the $p$-side boundary of the space-charge region, which can be obtained from the usual quasiequilibrium condition of constant chemical potentials (applied to nondegenerate statistics) from $x_{p}$ to $x_{n}$, is $\alpha_{p}=\left(\alpha_{0}+\alpha_{n}\right) /\left(1+\alpha_{0} \alpha_{n}\right)$. The nonequilibrium minority carrier density at $x=x_{p}$ is 
$n\left(x_{p}\right)=n_{0}\left(1+\alpha_{0} \alpha_{n}\right) \exp \left(V / V_{T}\right)$. With these boundary conditions the carrier and spin profiles in the magnetic $p$ region are

$$
\begin{gathered}
\frac{n(x)}{n_{0}}=1+\frac{\sinh \left(x / L_{n}\right)}{\sinh \left(x_{p} / L_{n}\right)}\left[e^{V / V_{T}}\left(1+\alpha_{n} \alpha_{0}\right)-1\right], \\
\frac{s(x)}{s_{0}}=\frac{n(x)}{n_{0}}+\frac{\sinh \left(x / L_{s p}\right)}{\sinh \left(x_{p} / L_{s p}\right)} \frac{\alpha_{n}}{\alpha_{0}}\left(1-\alpha_{0}^{2}\right) e^{V / V_{T}} .
\end{gathered}
$$

Having the profiles, we calculate the current in the magnetic $p$ - $n$ junction. We distinguish equilibrium-spin electron $J_{n}$ and hole $J_{p}$ currents, and nonequilibriumspin-induced current $J_{n}^{\prime}$, so that the total charge current is $J=J_{n}+J_{p}+J_{n}^{\prime}$. The individual contributions are

$$
\begin{gathered}
J_{n}=q \frac{D_{n}}{L_{n}} \frac{n_{i}^{2}}{N_{a}} \cosh \left(\frac{\zeta}{V_{T}}\right) \operatorname{coth}\left(\frac{x_{p}}{L_{n}}\right)\left(e^{V / V_{T}}-1\right), \\
J_{p}=q \frac{D_{p}}{L_{p}} \frac{n_{i}^{2}}{N_{d}} \operatorname{coth}\left(\frac{L-x_{n}}{L_{p}}\right)\left(e^{V / V_{T}}-1\right), \\
J_{n}^{\prime}=q \frac{D_{n}}{L_{n}} \frac{n_{i}^{2}}{N_{a}} \operatorname{coth}\left(\frac{x_{p}}{L_{n}}\right) \sinh \left(\frac{\zeta}{V_{T}}\right) \alpha_{n} e^{V / V_{T}} .
\end{gathered}
$$

Figure 4 shows how well this analytical model compares with our numerical calculation. The small discrepancy is caused by the neglect of the recombination processes in the space-charge region. The model breaks down at large bias, where the Shockley approximation is not valid [6]. We note that if the spin is injected by the bias contact at $x=L$, one needs to consider a model in which $\alpha(L)$ depends on $J$ (since it then obviously vanishes at $J=0$ ), and solve Eqs. (9)-(11) for $J$. Otherwise, the above equations describe either the case of an independent spin injection or the region of $J$ where $\alpha(L)$ is independent of $J$.

The large exponential magnetoresistance effect, $J \sim$ $\exp \left(|\zeta| / V_{T}\right)$ at large $|\zeta|$ (or $B$ ), comes from the increase of the minority electron population with $|\zeta|$. However, once a nonequilibrium spin population (finite $\alpha_{n}$ ) is maintained in the space-charge region, a giant magnetoresistance (GMR) should be observed, $J \sim \exp \left(\zeta / V_{T}\right)$ at large $|\zeta|$ and $\alpha_{n} \rightarrow$ \pm 1 . The GMR coefficient is then $\exp \left(2 \zeta / V_{T}\right)$. In addition to sensing $B$, these effects can be used for an allelectrical probing of the injected spin polarization $\alpha(L)$ and spin-diffusion length $L_{s n}$, as they both determine $\alpha_{n}$ (a possible device scheme is proposed in Fig. 1D). The key to employing the large magnetoresistance effects in spintronics is the development of materials with large $g$ factors. Even for $g \approx 100$, the GMR coefficient at $T=100 \mathrm{~K}$ and $B=1 \mathrm{~T}$ is about $\exp (0.65)$, which is close to $200 \%$. A magnetic impurity that would give $g \approx 500$ would yield $2500 \%$ (300\% at room temperature). The device potential of magnetic $p-n$ junctions is enormous, but to take full advantage we need materials with large $g$ factors at room temperature [10].

Equations (9)-(11) reveal another interesting phenomenon, a spin-voltaic effect. The current $\left(J_{n}^{\prime}\right)$ stays finite even for $V=0$, if $\alpha_{n} \neq 0$. A current flows at zero bias.
This is an analogue of the photovoltaic effect, where a reverse current flows if carriers are photogenerated in the space-charge region. In the spin-voltaic effect both reverse and forward currents can flow, depending on the relative orientation of $B$ and $\alpha_{n}$; the device can function as a spin valve. The physics of the spin-voltaic effect is that nonequilibrium spin in the space-charge region disturbs the balance between the generation and recombination currents (Fig. 1C). If $\zeta>0$, and more spin up electrons are present at $x_{n}\left(\alpha_{n}>0\right)$, the barrier for them to cross the region is smaller than the barrier for the spin down electrons, so more electrons flow from $n$ to $p$ than from $p$ to $n$, and positive charge current results. If $\alpha_{n}<0$, the current is reversed.

In summary, we have studied bipolar spin-polarized transport in magnetic/nonmagnetic $p$ - $n$ junctions. We predict novel phenomena of exponential and giant magnetoresistance, and of spin-voltaic effect, if a nonequilibrium spin is added into the junction. We have demonstrated that no spin injection or extraction is possible under low bias, and that only nonequilibrium spin can be transferred through the space-charge region. Finally, we have introduced a modified Shockley model which explains our numerical findings.

This work was supported by DARPA and the U.S. ONR.

*Present address: Institute for Theoretical Physics, KarlFranzens University, $8010 \mathrm{Graz}$, Austria.

[1] S. Das Sarma, J. Fabian, X. Hu, and I. Žutić, IEEE Trans. Magn. 36, 2821 (2000); Superlattices Microstruct. 27, 289 (2000); Solid State Commun. 119, 207 (2001).

[2] R. Fiederling, M. Kleim, G. Reuscher, W. Ossau, G. Schmidt, A. Waag, and L. W. Molenkamp, Nature (London) 402, 787 (1999); Y. Ohno, D. K. Young, B. Beschoten, F. Matsukura, H. Ohno, and D. D. Awschalom, Nature (London) 402, 790 (1999); B. T. Jonker, Y. D. Park, B. R. Bennett, H. D. Cheong, G. Kioseoglou, and A. Petrou, Phys. Rev. B 62, 8180 (2000).

[3] P. R. Hammar, B. R. Bennett, M. J. Yang, and M. Johnson, Phys. Rev. Lett. 83, 203 (1999).

[4] S. Datta and B. Das, Appl. Phys. Lett. 56, 665 (1990); M.E. Flatte and G. Vignale, Appl. Phys. Lett. 78, 1273 (2001).

[5] I. Žutić, J. Fabian, and S. Das Sarma, Phys. Rev. B 64, 121201 (2001); Appl. Phys. Lett. 79, 1558 (2001).

[6] S. Tiwari, Compound Semiconductor Device Physics (Academic Press, San Diego, 1992).

[7] M. Johnson and R. H. Silsbee, Phys. Rev. B 35, 4959 (1987); J.-E. Wegrowe, Phys. Rev. B 62, 1067 (2000).

[8] For ferromagnetic semiconductors $\zeta$ and carrier spin density need to be evaluated self-consistently.

[9] Term minority (majority) refers to the relative population of electrons and holes.

[10] At low temperatures $\mathrm{Cd}_{0.95} \mathrm{Mn}_{0.05}$ Se has $g>500$ : T. Dietl, in Handbook of Semiconductors, edited by T. S. Moss and S. Mahajan (North-Holland, New York, 1994), Vol. 3, p. 1279. 